\title{
Appearance of T Cell Subpopulations in the Chicken and Embryo Retina
}

\author{
Tomohiro IMAGAWA ${ }^{1)}$, Hiroshi KITAGAWA ${ }^{2)}$ and Masato UEHARA ${ }^{1)}$ \\ ${ }^{1)}$ Department of Veterinary Anatomy, Faculty of Agriculture, Tottori University, Tottori 680-8553 and ${ }^{2)}$ Department of Life Science, \\ Graduate School of Science and Technology, Kobe University, Kobe 657-8501, Japan
}

(Received 21 June 2002/Accepted 18 September 2002)

ABSTRACT. Under pathological conditions such as autoimmune encephalomyelitis or autoimmune uveoretinitis, many T cells infiltrate the central nervous system (CNS) and retina. Even in normal condition, a small number of T cells are detected in the CNS. However the characteristics of the $\mathrm{T}$ cells are not defined. To investigate the $\mathrm{T}$ cell characteristics in a healthy retina, the chicken and the embryo were observed by morphological and immunohistochemical methods. In the chicken retina, T cells were regularly detected, and the main subset was $\mathrm{CD}-8^{+} / \gamma \delta$ cells. Developmentally, CD positive cells appeared on embryonic day 13 , and the constituent $\mathrm{T}$ cell repertoires became the same as in the chicken by embryonic day 17. Many T cell repertoires were detected on embryonic day 15 and 16 . The present results confirm that the retina receives an immunological surveillance by $\mathrm{T}$ cells. The composition of $\mathrm{T}$ cells in retina is constructed after embryonic day 17. Many ganglion cells die in embryonic days 15 and 16. So the T cell subsets in these periods may involve in autoimmune diseases.

KEY WORDS: CD, development, retina, $\mathrm{T}$ cell receptor, $\mathrm{T}$ cell subset.

J. Vet. Med. Sci. 65(1): 23-28, 2003

It has been considered that the central nervous system (CNS) and retina are immunologically privileged sites [3]. This concept is supported by the evidences that lymphoid cell lineage is not found under normal conditions, and that allograft rejection fails in the CNS [19]. However, immune responses within the CNS are found in some pathological conditions such as multiple sclerosis [21] and its animal model, experimental autoimmune encephalomyelitis (EAE) [28]. In these diseases, a considerable number of activated T lymphocytes infiltrate the CNS. Similar autoimmune diseases can be induced by injecting a chicken with myelin basic protein (MBP) [25]. The underlying mechanisms have been investigated, and the results indicated that the activated $\mathrm{T}$ cells break down the blood-brain barrier [15, 17], and that the T lymphocytes in multiple sclerosis lesions are $\mathrm{CD}-3^{+} \mathrm{CD}-8^{+} \mathrm{T}$ cells [16]. Developmentally and histologically the retina is an extension of the CNS. The same pathological features have been reported in the eye as in the CNS such as experimental autoimmune uveoretinitis (EAU), which is a T-cell mediated autoimmune disease [12]. The retinal glial cells express immune-associated surface markers [8]. The T lymphocytes in EAU are $\mathrm{CD}-4^{+} / \mathrm{T}$ cell receptor $(\mathrm{TCR})^{+} \mathrm{T}$ cells [7].

In immune reactions, $\mathrm{T}$ cells play important roles and are classified into repertoires based on their different functions such as antigen recognition, induction of antibody production (helper $\mathrm{T}$ cell) and cytotoxisity (cytotoxic T cell). In organs other than the CNS, T lymphocyte recirculation is known to play a critical role in immunological surveillance. The T lymphocytes develop and mature in the thymus, then enter the circulation and the peripheral organs after maturation. The generation of an immune response is initiated by antigen presentation, followed by recognition by antigenspecific T cells. As for immune responses in the CNS, the ability of antigen presentation is detected in the glial cells (astrocytes and microglia) and the endothelial cells of the retina and brain $[17,30,32]$. Since the activated $\mathrm{T}$ cells infiltrate into the CNS or retina after MBP injection, the antigen-specific $\mathrm{T}$ cells should be present in the normal animal. However, many authors detected a small number of $\mathrm{T}$ cells in the CNS of healthy control animals [13, 14, 21, 28], but the features of those $\mathrm{T}$ cells are not described. Thus, some fundamental questions about $\mathrm{T}$ cells in the CNS still remain. Where and how are naive $T$ cells initiated against self-antigen such as MBP or retinal antigen of the CNS or retina, and how do the activated $\mathrm{T}$ cells initially recognize the antigen in the CNS?

Most $\mathrm{T}$ cells mature in the thymus, and are selected by their reactivity against major histocompatibility complex molecules and antigenic peptides during the ontogenetic period. The $\mathrm{T}$ cells with strong self-antigen reactivity are eliminated by negative selection, but not those with weak reactivity [29]. During ontogeny or after birth, T cells emigrate into the peripheral tissues from the thymus. Such emigration patterns are well defined in the chicken, in which the thymocytes colonize peripheral organs in discrete waves [11]. Those T cells that escape negative selection in the thymus and/or show weak reactivity may be candidates for effecter cells in CNS inflammatory diseases.

To investigate the $\mathrm{T}$ cell characteristics in healthy animals and their embryos may provide clues to understand the processes of autoimmune diseases in the CNS and retina. For this purpose, the chicken is a useful subject because of the ease in handling the embryos and the wealth of information about $\mathrm{T}$ cells as described above. In the present study, we investigated the appearance of T cells, and detected their subpopulations and occurrence in the chicken and the embryonic retina. 


\section{MATERIALS AND METHODS}

White Leghorn chickens and their embryos kept in our laboratory were used. Three chickens more than 2 months old were sacrificed by cervical exsanguination under anesthesia with diethyl ether. Three embryos from each embryonic day from 8 to 17 (E 8 to E 17) were decapitated. The eyes were removed and cut at the equator. The caudal hemispheres were fixed in cold periodate-lysine-paraformaldehyde solution buffered by $0.01 \mathrm{M}$ phosphate ( $\mathrm{pH}$ 7.4). The specimens were embedded in OCT compound and frozen in liquid nitrogen. Eight $\mu \mathrm{m}$ cryosections cut on a longitudinal plane containing the optic nerve head were prepared and washed in $0.1 \mathrm{M}$ phosphate buffer ( $\mathrm{pH} 7.4$; PB). After incubation in $0.3 \% \mathrm{H}_{2} \mathrm{O}_{2}$ and $99 \%$ methanol for $1 \mathrm{hr}$, the sections were treated by the peroxidase-anti-peroxidase (PAP) method. In brief, the sections were dipped in primary antiserum for $18 \mathrm{hr}$ at $4^{\circ} \mathrm{C}$, followed by secondary antiserum and PAP for $1 \mathrm{hr}$ at room temperature respectively. Between incubations, the sections were washed 3 times in $\mathrm{PB}$ and were finally incubated with 3,3'-diaminobenzidine containing $0.03 \% \mathrm{H}_{2} \mathrm{O}_{2}$. Anti chicken CD-3, -4 and -8 , and anti chicken TCR-1, -2 and -3 mouse monoclonal antibody (Southern Biotechnology Associates, Inc., Birmingham, U.S.A.) were used as the primary antiserum diluted 50 times in PB. Anti-mouse IgG goat serum as the secondary antiserum was purchased from Seikagaku Corporation (Tokyo, Japan), and mouse peroxidase-anti-peroxidase complex was from Jackson Immunoresearch Lab., Inc.(Pennsylvania, U.S.A.).

The eyes of the same chickens and embryos as above were fixed in a $2 \%$ paraformaldehyde and $2.5 \%$ glutaraldehyde mixture in $\mathrm{PB}$ for $2 \mathrm{hr}$ at $4^{\circ} \mathrm{C}$. The specimens were cut into small pieces, and postfixed with $1 \% \mathrm{OsO}_{4}$ for $1.5 \mathrm{hr}$ at room temperature. After dehydration with a series of alcohol, the pieces were embedded in epok-812. Semithin sections were stained with $0.1 \%$ toluidine blue, and ultrathin sections were contrasted with uranyl acetate and lead citrate and viewed with a transmission electron microscope (JEM 1210, JEOL, Tokyo, Japan).

\section{RESULTS}

Under light microscopy of toluidine blue stained sections, lymphoid cells were found in the nerve fiber layer, and they were small and contained dark round or oval nuclei (Fig. 1). The cells tend to locate at a vitreous site, but some were observed near the ganglion cells. Immunohistochemically, the number of cells with CD molecules and TCR was smaller than that of lymphoid cells in the toluidine blue stained sections. One to three positive cells were detected in each section, which was a plane containing the optic nerve head and anterior end of the retina.

In chicken retina, CD-3 positive lymphoid cells were found in all specimens (Fig. 2). The positive cells were mainly found on the vitreous side in the nerve fiber layer. In the embryonic retina, no positive reactions were detected

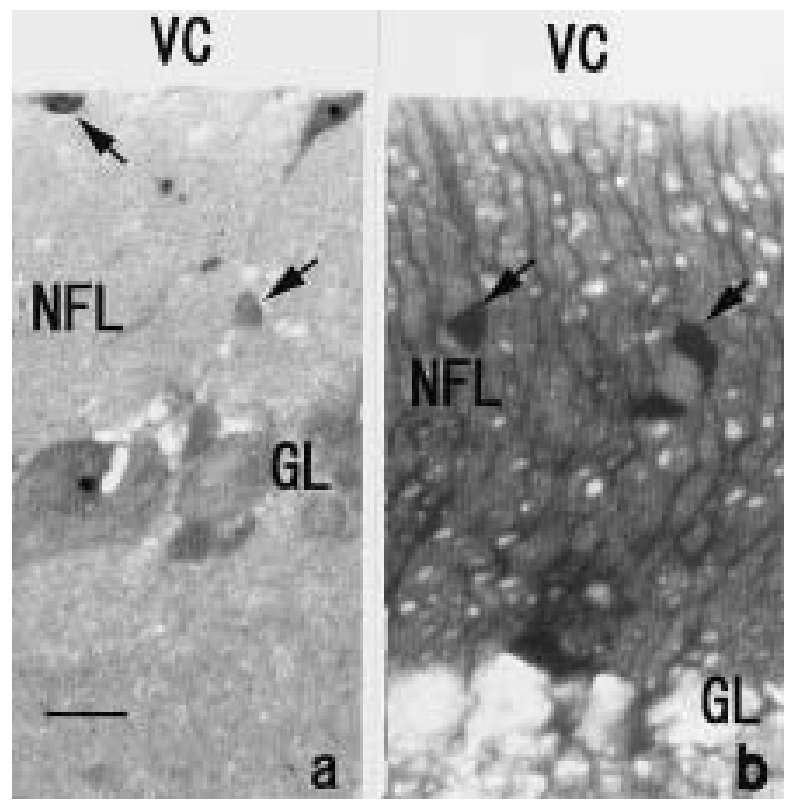

Fig. 1. Toluidine blue-stained semi-thin sections of the retina on E 14 (a) and chicken (b). Arrows indicate lymphoid cells in the nerve fiber layer (NFL). GL: ganglion cell layer. VC: vitreous chamber. Bar $=10 \mu \mathrm{m}$.

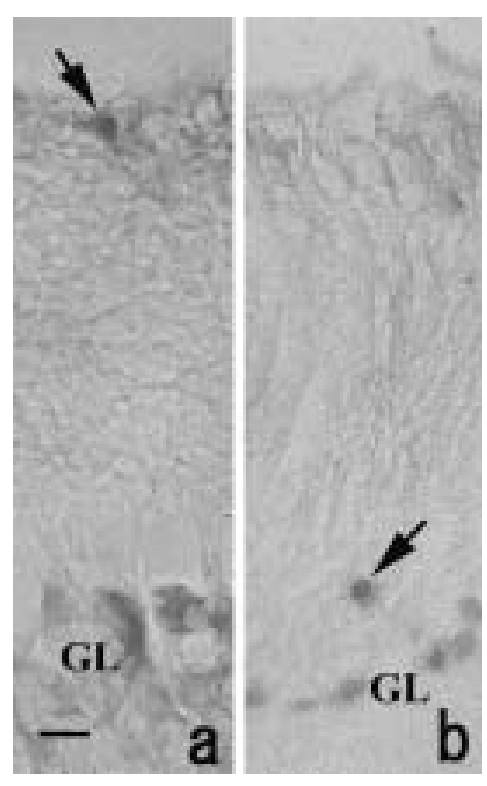

Fig. 2. Immunohistochemical reactions for CD-3 on E 13 (a) and chicken (b). Arrows show CD-3 positive cells in the nerve fiber layer. GL: ganglion cell layer. Bar $=10 \mu \mathrm{m}$.

before $\mathrm{E} 10$. The $\mathrm{CD}-3$ positive cells were present in all periods after 12 days. The reactivity for $\mathrm{CD}-3$ was weak on E 11 and 12, and became strong after E 13. After 17 days of 


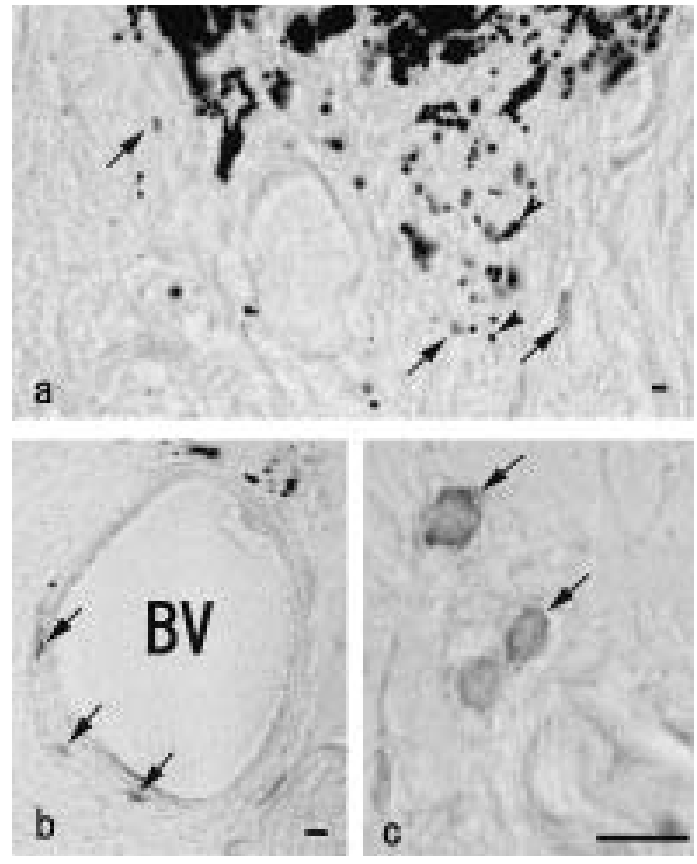

Fig. 3. CD-3 positive cells (arrows) in the optic nerve head. Small dark dots (arrow heads) are pigment granules originating from the pecten (a). Around a blood vessel (BV) in the optic nerve head many CD- 8 positive cells (arrows) appear (b). The CD-3 positive cells (arrows) are round or oval shape with peripheral positive reactions (c). Bars $=10 \mu \mathrm{m}$.

incubation, the number and reactivity of CD-3 positive cells were the same as those of chickens (Fig. 2). Positive cells in the optic nerve head and in the nerve fiber layer around the head were relatively numerous (Fig. 3).

CD-8 positive cells were also detected at the same site as CD-3 positive cells, but in fewer numbers (Fig. 4). CD-4 positive cells were rarely observed in the nerve fiber layer of chicken retina. In the same embryonic periods as the CD-3, CD- 8 positive cells were observed, but CD-4 positive cells were only detected after E 16 (Fig. 4). As for T cell receptors, only TCR-1 positive reactions were detected in chicken retina (Fig. 5). There were no positive reactions in other layers of the retina. Developmentally, the TCR expressions were later than the CD molecules. TCR-1 was detected after E 15. TCR-2 positive cells were appeared after E 15, and the reactions were not observed at E 17 . Quite a few TCR3 positive cells were detected only on E 15 .

The appearances of CD molecules and TCRs in chicken and embryo retina were summarized in Table 1.

Under transmission electron microscopy, characteristic lymphocytes were detected in retina (Fig. 6). The cells were represented by chromatin rich small nuclei, small amount of cytoplasm containing a few cytoplasmic organelles, large round mitochondria, and a small number of rough endoplasmic reticula. After E 15 in the embryo and chicken retina, lysosomal granules with high electron density were fre-

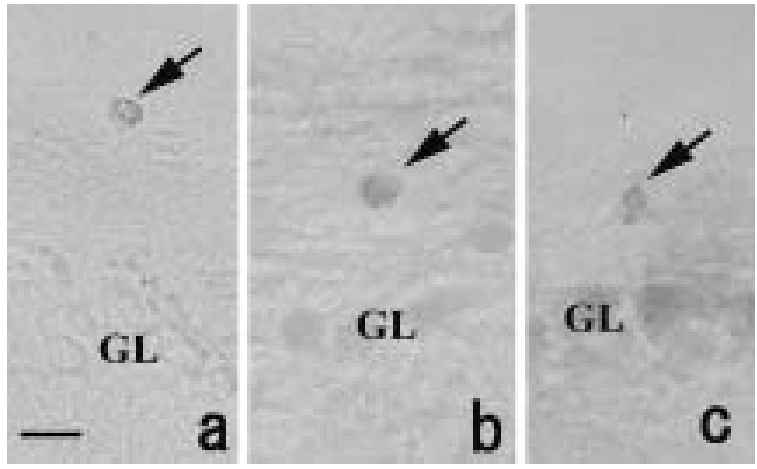

Fig. 4. Immunohistochemical reactions for CD-8 on E 17 (a) and chicken (b), and CD-4 on E 16 (c). Arrows show CD-positive cells. GL: ganglion cell layer. Bars $=10 \mu \mathrm{m}$.

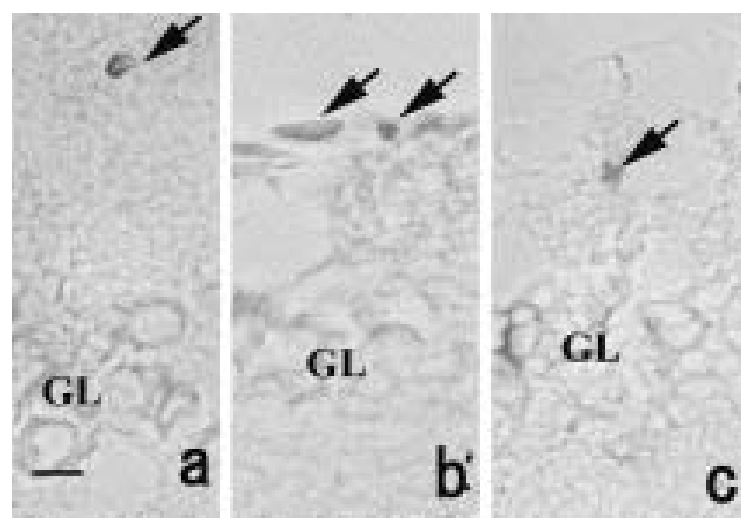

Fig. 5. Immunohistochemical reactions for TCR-1 on E 16 (a) and chicken (b), and TCR-2 on E 15 (c). Arrows show TCR positive cells. GL: ganglion cell layer. $\operatorname{Bar}=10 \mu \mathrm{m}$.

Table 1. Appearance of CD molecules (CD-3, -4 and -8 ) and T cell receptors (TCR-1, -2 and -3 ) in chicken and embryonic retina

\begin{tabular}{lcccccccccc}
\hline & $* 8$ & to 10 & 11 & 12 & 13 & 14 & 15 & 16 & 17 & chicken \\
\hline CD-3 & - & \pm & \pm & + & + & + & + & + & + \\
CD-4 & - & - & - & - & - & - & + & + & \pm \\
CD-8 & - & - & - & - & + & + & + & + & + & + \\
TCR-1 & - & - & - & - & - & + & + & + & + \\
TCR-2 & - & - & - & - & - & + & + & - & - \\
TCR-3 & - & - & - & - & - & \pm & - & - & - \\
\hline
\end{tabular}

*: numbers indicate incubation days of embryo.

-: not detected in all specimens of each embryonic day and chicken. \pm : detected in some specimens of each embryonic day and chicken.

+ : detected in all specimens of each embryonic day and chicken.

quently observed in the cytoplasm of the lymphocytes.

\section{DISCUSSION}

Recent observations suggest that the central nervous tissue is not an immunologically privileged site. There is a 

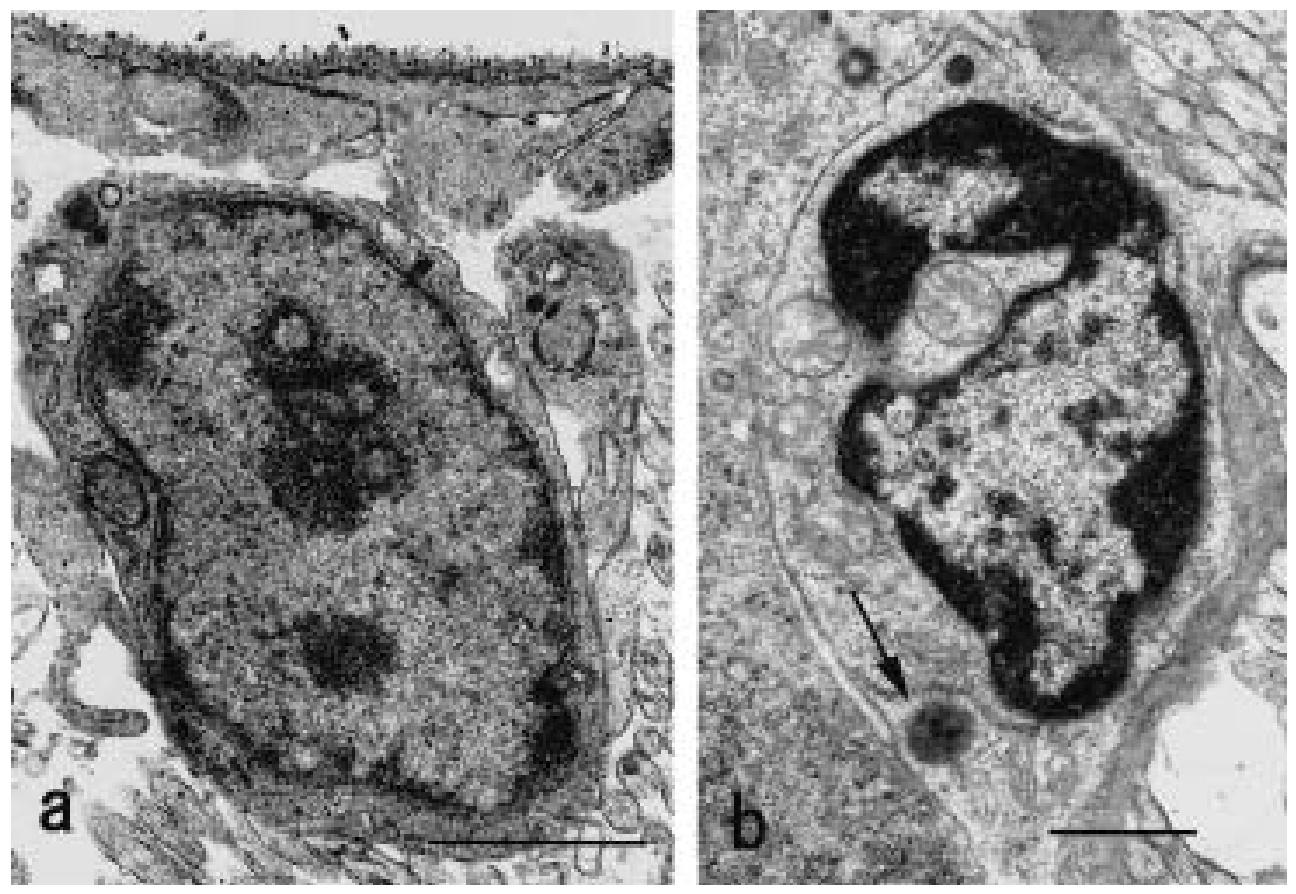

Fig. 6. Electron micrographs of lymphocytes in the nerve fiber layer on E 12 (a) and chicken (b). Arrow indicates the electron dense lysosomal granule.

hypothesis that immature $\mathrm{T}$ cells can enter into the CNS [31]. Some authors directly demonstrated T cell entry into the CNS [13] and the retina [20] of the rat. They also showed that $\mathrm{T}$ cells appear even under normal conditions. Although the repertoires of $\mathrm{T}$ cells are defined in some pathological lesions, no information exists about $\mathrm{T}$ cells under normal conditions and developmental period. The present study showed that lymphocytes were morphologically detected, and that immunohistochemically CD-3 positive cells were apparently observed in both the chicken and the embryo retina. These results show that $\mathrm{T}$ cells regularly circulate in the normal retina of the chicken and embryo, although the number in small.

During embryonic periods, at first the only $\mathrm{T}$ cell detected was $C D-3$ positive on $E 11$. In these early periods (E 11 and 12 ), the CD-3 positive cells were not observed in all specimens and their immunohistochemical reactions were weak, suggesting that the T cells on $\mathrm{E} 11$ and 12 may be immature. In the chicken and embryos after E 13, CD-3 positive cells with strong reactivity were clearly observed. It is reported that the $\mathrm{T}$ cell progenitors colonize the thymus and peripheral organs in 3 discrete waves; E 6 to 8, E 12 to 14 and E 18 to 21 in the thymus [11]. The T cells appearance period (E 13) in the retina corresponds to the time of the second wave of progenitors in the thymus and is earlier than the first wave in the spleen and intestine (E 18 to 20).

In the avian immune system, large numbers of $\mathrm{T}$ cells express the $\gamma \delta \mathrm{T}$ cell receptor [26], and two-thirds of the T cells in the spleen and intestinal epithelium express CD-8 [4]. The present immunohistochemical results for the chicken showed that the $\mathrm{T}$ cell receptor was only TCR-1 (specific for $\gamma \delta \mathrm{T}$ cell receptor), and that CD-8 positive cells were observed more frequently than CD-4 positive cells. It is suggested that the $\mathrm{T}$ cells in chicken retina are mainly $\mathrm{CD}-8^{+} / \gamma \delta \mathrm{T}$ cells similar to those in the intestine and spleen.

Developmentally, CD-8 positive cells were detected at the same time as CD-3 expression after E 13. The T cell receptors were observed after $\mathrm{E} 15$ in the retina. This period of $\mathrm{T}$ cell receptor expression is similar to that in the other organs of the embryo $[5,9,10]$. The $\gamma \delta \mathrm{T}$ cell receptor was regularly observed after E 15 , while the $\alpha \beta$ (TCR-2 and -3) receptors were detected only on E 15 and 16 . It seems that the $\mathrm{CD}-8^{+} / \gamma \delta$ cells are also the main $\mathrm{T}$ cell subset in the embryonic retina, and that their composition after E 17 is identical to the chicken. CD-4 positive cells appeared only on E 16 and 17, which was later than CD-8 positive cells. Since no $\mathrm{CD} 4^{+} / \gamma \delta \mathrm{T}$ cells have been described in the chicken [2], the CD- $4^{+}$cells in the embryonic retina are CD $-4^{+} / \alpha \beta \mathrm{T}$ cells. The $\alpha \beta$ receptors were detected on E 15 and 16 , not in E 17 embryo and chicken retina. We cannot offer any adequate explanation for this. The time of the first wave of $\alpha \beta$ $\mathrm{T}$ cell progenitors colonizing the spleen and the intestine is around $\mathrm{E} 18$, and the second and third waves continue after hatch [11]. Thus, the CD-4+ $\mathrm{T}$ cells in the retina on E 17 may be immature $\mathrm{T}$ cells originating from the first thymocyte emigration wave.

During E 15 and 16, T cell repertoires were enriched in the retina. In addition to the $\mathrm{CD}-8^{+} / \gamma \delta$ cells, possible combinations of $\mathrm{T}$ cells during this period were $\mathrm{CD}-8^{+} / \alpha \beta$ on $\mathrm{E}$ 15 , and $\mathrm{CD}-8^{+} / \alpha \beta, \mathrm{CD}-4^{+} / \alpha \beta$ and probably including dou- 
ble positive $\left(\mathrm{CD}-4^{+} / \mathrm{CD}-8^{+}\right)$cells on $\mathrm{E} 16$. The $\mathrm{CD}-8^{+} / \alpha \beta \mathrm{T}$ cell function is cytotoxic, and CD $-4^{+} / \alpha \beta$ cell is helper T cell producing cytokines [2]. Electronmicroscopical observations showed that some of the lymphocytes in embryo retina possessed lysosomal granules, which indicate the cytotoxic function of the cells. It is possible that these $\mathrm{T}$ cell repertoires during E 15 and 16 are involved in ganglion cell death and in removing the cell debris. Developmentally, excess ganglion cells are dead by around E 16 [24]. It is tempting to speculate that some of the $\mathrm{CD}-4^{+} / \alpha \beta$ cells on $\mathrm{E} 16$ may activate to the ganglion cell elements including MBP and provide help to other kinds of T cells $\left(\mathrm{CD}-8^{+} / \gamma \delta\right.$ and $\mathrm{CD}-8^{+} /$ $\alpha \beta$ ). A certain percentage of the activated $\mathrm{T}$ cells may remain as memory $\mathrm{T}$ cells, which possess reactivity to MBP in adulthood.

In general, lymphocytes proliferate and mature in the thymus. According to the clonal selection theory [1], a great diversity of lymphocytes is generated, and the self-antigen reacting lymphocytes are eliminated by negative selection in the thymus before migration to the peripheral organs [29]. On the other hand, the extrathymic development of $\gamma \delta$ T cell has been reported in the intestine of the mouse [23]. Cooper et al. [6] reported that the $\gamma \delta \mathrm{T}$ cells migrate quickly from the thymus without undergoing clonal expansion in the chicken. The present study indicated that cells with weak immunoreactivity against CD-3 appeared at early periods (E 11 and 12) in the retina. Some of these immature T cells are thought to be naive lymphocytes capable of recognizing self-peptides.

The $\mathrm{T}$ lymphocyte infiltration of the brain and retina occurs in some pathological conditions, e.g., multiple sclerosis, experimental autoimmune encephalomyelitis (EAE) [22] and experimental autoimmune uveoretinitis (EAU) [21]. In both the EAE and the EAU, activated T cells infiltrate via the blood vessels into the CNS and retina. During the pathogenesis of EAU in the rat, endothelial cells of the retinal blood vessels express class II MHC antigen [17] and show morphological changes [18]. Unlike rat retina, the chicken retina is composed of avascular tissue, so that there are no blood vessels on its vitreous surface. The retina is nurtured by blood vessels in choroids and in the optic nerve head, which supply blood to the pecten. Our present observations revealed many lymphocytes around the optic nerve head, and immunohistochemical positive reactions were also detected. This suggests that the $\mathrm{T}$ cells in the retina emigrate through the blood vessels in the optic nerve head.

\section{REFERENCES}

1. Ada, G.L. and Nossal, G. 1987. The clonal-selection theory. Sci. Am. 257: 50-57.

2. Arstila, T.P. 1996. T cell subsets and the activation of $\gamma \delta \mathrm{T}$ cells. pp. 71-77. In: Immunology and Developmental Biology of the Chicken (Vainio, O. and Imhof, B.A. eds.), Springer, Berlin.

3. Barker, C.F. and Billingham, R.E. 1977. Immunologically privileged sites. Adv. Immunol. 25: 1-54.

4. Bucy, R.P., Chen, C.H., Cihak, J., Losch, U. and Cooper, M.D.
1988. Avian T cells expressing $\gamma \delta$ receptors localize in the splenic sinusoids and the intestinal epithelium. J. Immunol. 141: 2200-2205.

5. Chen, C.H., Cihak, J., Losch, U. and Cooper, M.D. 1988. Differential expression of two T cell receptors, TCR 1 and TCR 2, on chicken lymphocytes. Eur. J. Immunol. 18: 539-543.

6. Cooper, M.D., Chen, C.H., Bucy, R.P. and Thompson, C.B. 1991. Avian T cell ontogeny. Adv. Immunol. 50: 87-117.

7. Dick, A.D., McMenamin, P.G., Korner, H., Scallon, B.J., Ghrayeb, J., Forrester, J.V. and Sedgwick, J.D. 1996. Inhibition of tumor necrosis factor activity minimizes target organ damage in experimental autoimmune uveoretinitis despite quantitatively normal activated $\mathrm{T}$ cell traffic to the retina. Eur. J. Immunol. 26: 1018-1025.

8. Drescher, K.M. and Shittum-Hudson, J.A. 1996. Modulation of immune-associated surface markers and cytokine production by murine retinal glial cells. J. Neuroimmunol. 64: 71-81.

9. Dunon, D. and Imhof, B.A. 1996. T cell migration during ontogeny and T cell repertoire generation. pp. 79-94. In: Immunology and Developmental Biology of the Chicken (Vainio, O. and Imhof, B.A. eds.), Springer, Berlin.

10. Dunon, D., Cooper, M.D. and Imhof, B.A. 1993. Migration patterns of thymus-derived $\gamma \delta$ T cells during chicken development. Eur. J. Immunol. 23: 2545-2550.

11. Dunon, D., Schwager, J., Dangy, J.-P., Cooper, M.D. and Imhof, B.A. 1994. T cell migration during development: homing is not related to TCR V $\beta 1$ repertoire selection. EMBO J. 13: $808-815$.

12. Greenwood, J., Howes, R. and Lightman, S. 1994. The bloodretinal barrier in experimental autoimmune uveoretinitis: leukocyte interactions and functional damage. Lab. Invest. 70: 3952.

13. Hickey, W.F., Hsu, B.L. and Kimura, H. 1991. T-lymphocyte entry into the central nervous system. J. Neurosci. Res. 28 : 254-260.

14. Hickey, W.F., Lassmann, H. and Cross, A.H. 1997. Lymphocyte entry and the initiation of inflammation in the central nervous system. pp. 200-225. In: Immunology of the Nervous System (Keane, R.W. and Hickey, W.F. eds.), Oxford University Press, New York.

15. Hu, P., Pollard, J.D. and Chan-Ling, T. 2000. Breakdown of the blood-retinal barrier induced by activated $\mathrm{T}$ cells of nonneural specificity. Am. J. Pathol. 156: 1139-1149.

16. Jewtoukoff, V., Lebar, R. and Bach, M.-A. 1989. Oligodendrocyte-specific autoreactive T cells using an alpha/beta T-cell receptor kill their target without self restriction. Proc. Natl. Acad. Sci. U.S.A. 86: 2824-2828.

17. Lightman, S. 1988. Immune mechanisms in autoimmune ocular disease. Eye 2: 260-266.

18. McMenamin, P.G., Forrester, J.V., Steptoe, R.J. and Dua, H.S. 1992. Ultrastructural pathology of experimental autoimmune uveitis. Quantitative evidence of activation and possible high endothelial venule-like changes in retinal vascular endothelium. Lab. Invest. 67: 42-55.

19. Medawar, P.B. 1948. Immunity of homologous grafted skin. III. The fate of skin homografts transplanted to the brain, to subcutaneous tissue and to the anterior chamber of the eye. $\mathrm{Br}$. J. Exp. Pathol. 29: 58-69.

20. Prendergast, R.A., Iliff, C.E., Coskuncan, N.M., Caspi, R.R., Sartani, G., Tarrant, T.K., Lutty, G.A. and McLeod, D.S. 1998. $\mathrm{T}$ cell traffic and the inflammatory response in experimental autoimmune uveoretinitis. Invest. Ophthalmol. Vis. Sci. 39: 754-762. 
21. Raine, C.S. 1994. The Dale E. McFarlin memorial lecture: the immunology of the multiple sclerosis lesion. Ann. Neurol. 36: S61-S72.

22. Raine, C.S., Cannella, B., Duijvestijn, A.M. and Cross, A.H. 1990. Homing to central nervous system vasculature by antigen-specific lymphocytes. II. Lymphocyte/endothelial cell adhesion during the initial stages of autoimmune demyelination. Lab. Invest. 63: 476-489.

23. Rocha, B., Vassalli, P. and Guy-Grand, D. 1992. The extrathymic T-cell development pathway. Immunol. Today 13: 449454.

24. Rogers, L.J. 1995. The Development of Brain and Behaviour in the Chicken, Cap International, Wallingford.

25. Rose, N.R. 1994. Avian models of autoimmune disease: lessons from the birds. Poult. Sci. 73: 984-990.

26. Sowder, J.T., Chen, C.H., Ager, L.L., Chan, M.M. and Cooper, M.D. 1988. A large subpopulation of avian T cells express a homologue of the mammalian $\mathrm{T} \gamma / \delta$ receptor. J. Exp. Med. 167: 315-322.
27. Thanos, S., Moore, S. and Hong, Y. 1996. Retinal microglia. Retinal Eye Res. 15: 331-361.

28. Trotter, J. and Steinman, L. 1984. Homing of Lyt-2 ${ }^{+}$and Lyt-2T cell subsets and B lymphocytes to the central nervous system of mice with acute experimental allergic encephalomyelitis. $J$. Immunol. 132: 2919-2923.

29. Von Boehmer, H. 1992. Thymic selection: a matter of life and death. Immunol. Today 13: 454-458.

30. Wang, Y., Calder, V.L., Lightman, S.L. and Greenwood, J. 1995. Antigen presentation by rat brain and retinal endothelial cells. J. Neuroimmunol. 61: 231-239.

31. Wekerle, H., Linington, H., Lassman, H. and Meyermann, R. 1986. Cellular immune reactivity within the CNS. Trends Neurosci. 9: 271-276.

32. Wekerle, H., Sun, D., Oropeza-Wekerle, R.L. and Meyermann, R. 1987. Immune reactivity in the nervous system: modulation of T-lymphocyte activation by glial cells.J. Exp. Biol. 132: 4357. 